\title{
Mesenchymal Stem-Cell Transplantation for Hypoxic-Ischemic Brain Injury in Neonatal Rat Model
}

\author{
JIN A. LEE, BEYONG IL KIM, CHRIS HYUNCHUL JO, CHANG WON CHOI, EE-KYUNG KIM, HAN-SUK KIM, \\ KANG-SUP YOON, AND JUNG-HWAN CHOI
}

\author{
Departments of Pediatrics [J.A.L.] and Orthopedics [C.H.J., K.-S.Y.], Seoul National University Boramae Hospital and Seoul National \\ University College of Medicine, Seoul, South Korea, 156-707; Department of Pediatrics [J.A.L., B.I.K., C.W.C., E.-K.K., H.-S.K., J.-H.C.], \\ Seoul National University College of Medicine, Seoul, South Korea, 110-744
}

\begin{abstract}
Neonatal hypoxic-ischemic brain injury (HIE) remains a major cause of neurologic disabilities. However, many experimental therapies have shown limited successes. We assessed whether human mesenchymal stem cells (MSCs) could be transplanted in the HIE rat brain to improve neurologic disabilities. P7 SD rats were either subjected to left carotid artery ligation and hypoxic exposure [hypoxia-ischemia (HI)] or sham operation and normoxic exposure (sham). On P10, rat pubs received either PKH26-labeled MSCs or buffer via intracardial injection, resulting in four experimental groups: sham-buffer, sham-MSC, HI-buffer, and HI-MSC. Cylinder test and accelerating rotarod test were performed 14, 20, 30, and $40 \mathrm{~d}$ after injection. Six weeks after injection, cresyl violet and double immunofluorescence staining were performed. MSCs were transplanted to the whole brain mainly after HI. Glial fibrillary acidic protein and OX42 were more abundantly colocalized with MSC than neuronal specific nuclear protein or myelin basic protein. There were no significant differences in the total amounts and cell types between the lesioned and nonlesioned hemisphere. The lesioned hemispheric volume was decreased after HI ( $p=0.012)$ but not restored by MSC. Neurologic performance was significantly impaired only on the cylinder test after HI $(p=0.034)$, and MSC transplants improved it $(p=0.010)$. These suggest MSC can be a candidate for the treatment of neonatal HIE. (Pediatr Res 67: 42-46, 2010)
\end{abstract}

$\mathrm{H}$ ypoxic-ischemic brain injury (HIE), a result of asphyxia at term, remains a major cause of neurologic disabilities. It occurs in $\sim 20$ of 1000 full-term infants. Between 20 and $50 \%$ of asphyxiated babies who exhibit HIE die during the newborn period. Of the survivors, up to $25 \%$ have permanent neuropsychological handicaps in the form of cerebral palsy, with or without associated mental retardation, learning disability, or epilepsy (1). However, except hypothermia, which has shown improvement only in mild to moderate HIE, many experimental therapies have met with limited success in the clinical environment (2).

Neural stem cells have been proposed as a useful treatment modality for the diseases of the CNS including neonatal HIE in animal models. However, their lack of accessibility and the inhomogeneity in neuronal differentiation of various neuro-

Received May 11, 2009; accepted August 29, 2009

Correspondence: Beyong Il Kim, M.D., Ph.D., Department of Pediatrics, Seoul National University Bundang Hospital, 166 Gumi-ro, Bundang-gu, Seongnam-si, Gyeonggi-do 463-707, South Korea; e-mail: beyil@snu.ac.kr

Supported by Seoul R\&BD Program No. 10548. This study was also partially supported by KT\&T Grant-in-Aid for neonatal research (SNUCH200601, SNUUCH200801). spheres limit their utility $(3,4)$. Mesenchymal stem cells (MSCs), as cell-based therapy offer a potential source of therapies for various rodent models and clinical trials of human diseases such as Parkinson's disease, Huntington's disease, myocardiac infarction, and stroke (5-9). However, limited data are available in neonatal HIE models. Guan et al. (10) reported that more MSCs were transplanted to the neonatal rat brain when MSCs were intraperitoneally injected $2 \mathrm{~h}$ after hypoxia-ischemia (HI) than when they were injected in the sham group. Yasuhara et al. reported that when they injected MSCs into the hippocampus of neonatal HI rats, MSC survived $14 \mathrm{~d}$ after post-transplantation and helped behavioral recovery. They did not assess the neural cell fate of MSC, but in their neurobehavioral assessment, they compared the neurobehavioral performance with that of the historical control (11). They also detected the i.v. grafts in the ischemic hippocampal area $14 \mathrm{~d}$ after transplantation, which improved behavioral recovery and exhibited the neuronal marker MAP2. However, they did not see whether the grafts can be differentiated into the other types of brain cells and they assessed the only short-term neurobehavioral outcome (12).

Therefore, this study was conducted to determine whether human bone marrow-derived MSCs could be effectively transplanted to the neonatal rat HI brain via intracardiac injection and whether MSCs could reduce the brain infarction and improve the sensorimotor deficit if they were transplanted to the neonatal rat brain after HI.

\section{MATERIALS AND METHODS}

Isolation and purification of MSC. Fresh human bone marrow was harvested from patients who underwent orthopedic surgery after informed consent was obtained. MSCs were isolated as described by Friedenstein et al. (13). Briefly, mononuclear cells were isolated from Ficoll-Hypaque, separated bone marrow, and resuspended in human MSC medium consisting of $10 \%$ DMEM-low glucose (GIBCO; Grand island, NY), $10 \%$ fetal bovine serum (FBS, GIBCO), and $1 \%$ antibiotic-antimycotic solution (GIBCO). It was plated at $1 \times 10^{6}$ cells $/ 100 \mathrm{~cm}^{2}$. Cultures were maintained at $37^{\circ} \mathrm{C}$ in a humidified atmosphere containing $5 \% \mathrm{CO}_{2}$ for $3 \mathrm{Wk}$. After confluence, cells were trypsinized and replated at 5000 cells $/ 75 \mathrm{~cm}^{2}$. Every cell was cryopre-

Abbreviations: DAPI, 4',6'-diamidino-2-phenylindole dihydrochloride hydrate; FACS, fluorescence-activated cell sorting; GFAP, glial fibrillary acidic protein; HI, hypoxia-ischemia; HIE, hypoxic-ischemic brain injury; MBP, myelin basic protein; MSC, mesenchymal stem cell; NeuN, neuronal specific nuclear protein 
served at first passage and thawed, then recultured for transplantation. Fluorescence-activated cell sorting was performed for the identification of MSCs. MSCs were negative for CD14 (FITC-conjugated mouse anti-human CD14; BD Pharmingen, San Jose, CA), CD34 (PE-conjugated mouse anti-human CD34; BD Biosciences, San Jose, CA), and CD45 (FITC-conjugated mouse anti-human CD45, BD Biosciences). They expressed CD73 (PE-conjugated mouse anti-human CD73, BD Pharmingen) and CD105 (FITC-conjugated mouse anti-human CD105, Serotec; Kidlington, Oxford, UK).

Neonatal HI model. The Care of Experimental Animals Committee and the Internal Review Board of Seoul National University Hospital for human cell use approved all experimental procedures. One-d-old male SD rats were maintained at the Clinical Research Institute of Seoul National University Hospital. At the 7th day of life, rat pubs were anesthetized with an intraperitoneal injection of ketamine and xylazine hydrochloride, double ligated, and cut the left common carotid artery. The pups were returned to their dam for $2 \mathrm{~h}$ and exposed to $8 \%$ oxygen for $3.5 \mathrm{~h}$. Sham-operated animals were anesthetized, and a midline neck incision was made but the carotid artery was neither ligated nor cut.

MSC labeling. $\mathrm{PKH} 26$ staining was carried out for MSC labeling before transplantation. Briefly, after trypsinization, $2 \times 10^{7}$ MSCs were washed twice and resuspended into $1 \mathrm{~mL}$ of the dilution buffer provided by the manufacturer in the labeling kit. This cell suspension was mixed with an equal volume of the labeling solution containing $\mathrm{PKH} 26$ dye to reach the desired final concentrations. Labeling was allowed for $4 \mathrm{~min}$ at $25^{\circ} \mathrm{C}$ with periodical tapping of the tube. The reaction was terminated by the addition of $1 \mathrm{~mL}$ FBS. Cells were washed twice in $5 \mathrm{~mL}$ DMEM, and the labeling ratio was counted under a fluorescent microscope. Viability was assessed by the trypan blue exclusion method.

Transplantation. All rats were divided into four groups $72 \mathrm{~h}$ after $\mathrm{HI}$ or sham operation: the sham-buffer group $(n=10)$, sham-MSC group $(n=10)$, HI-buffer group $(n=12)$, and HI-MSC group $(n=12)$. Randomly selected rats slowly received $100 \mu \mathrm{L}$ suspension of red fluorescent dye PKH26 (Sigma Chemical Co.-Aldrich; St. Louis, MO)-labeled $1 \times 10^{6}$ MSCs or buffer (normal saline) for $1 \mathrm{~min}$ via an intracardiac injection. MSCs within five passages were grafted to the rats. Immunosuppressant was not used in any animals

Behavioral tests. Behavioral tests including the accelerating rotarod test and the cylinder test were performed 14, 20,30, and $40 \mathrm{~d}$ after injection. All behavioral tests were monitored by two individuals who were blinded to the rat treatment status.

In the accelerating rotarod test, the rats were placed in a rotarod cylinder, and the time the rats remained on the cylinder was measured. The speed of rolling was slowly increased from 4 to $40 \mathrm{rpm}$ within $5 \mathrm{~min}$. The trial was ended if the rat fell off the cylinder or gripped the device and spun around for two consecutive revolutions. The average duration was recorded with three measurements.

In the cylinder test, rats were placed in a $20-\mathrm{cm}$ diameter, $30-\mathrm{cm}$ high transparent acrylic cylinder. A mirror was placed behind the cylinder at an appropriate angle and their activity was videotaped for $5 \mathrm{~min}$. The number of wall contacts with forelimbs was counted. The wall contacts were classified as contact by contralateral forelimb (CF), ipsilateral forelimb (IF), or both forelimbs (BF). The percentage of the uses of $\mathrm{CF}$ and $\mathrm{BF}$ relative to the total number of contacts was calculated as follows: total percentage $=(\mathrm{CF}+$ $\mathrm{BF} / 2) /(\mathrm{CF}+\mathrm{BF}+\mathrm{IF}) \times 100$.

Histologic examination. Six wk after injection, each animal was reanesthetized with ketamine and xylazine and perfused through the heart with 100 $\mathrm{mL}$ of cold saline and $100 \mathrm{~mL}$ of $4 \%$ paraformaldehyde in $0.1 \mathrm{M}$ PBS. The brains were removed, postfixed for $1 \mathrm{~d}$ at $4^{\circ} \mathrm{C}$, and then cryoprotected with $30 \%$ sucrose for $24 \mathrm{~h}$. The tissues were embedded in OCT compound and frozen at $-70^{\circ} \mathrm{C}$. The brains were cut by cryostat (Leica CM 1900, Meyer Instruments, Inc.; Houston, TX) into 30- $\mu$ m sections. Each of the 12 sections was stained by cresyl violet to compare the hemispheric atrophy of the four experimental groups. The lesioned hemispheric volume was presented as a volume percentage of the lesioned hemisphere relative to the contralateral hemisphere using the Image $\mathbf{J}$ software (National Institutes of Health image software).

Quantification of histologic data. To determine how many MSCs were transplanted to the rat brain, transplanted cells were counted in each hemisphere in the six sections per brain corresponding to Figures 10, 20, 30, 40, 50 , and 60 of Paxino's rat brain atlas (14) by laser scanning confocal microscopy with the use of a laser-scanning confocal imaging system mounted onto a Zeiss microscope (LSM 510 META, Jena, Germany). The area was measured in the adjacent sections by cresyl violet staining using Image J. It was quantified in four rats per group. We took the pictures at the coronal sections including striatum and hippocampus in the sham-MSC and HI-MSC group using tile scan by laser scanning confocal microscope.
Double immunofluorescent staining was used to identify cells derived from MSCs with the free-floating method. Two independent investigators counted PKH26-positive cells with 4',6'-diamidino-2-phenylindole dihydrochloride hydrate (DAPI)-positive nuclei in each hemisphere. We also counted PKH26(+)-DAPI $(+)$ cells, which colocalized with neuronal specific nuclear protein (NeuN) (mouse monoclonal, Chemicon, Temecula, CA; 1:500), glial fibrillary acidic protein (GFAP) (rabbit polyclonal, Dako, Glostrup, Denmark; 1:1000), OX42 (mouse monoclonal, Chemicon; 1:400), and myelin basic protein (MBP) (mouse monoclonal, Upstate, NY; 1:1000). FITC-conjugated goat anti-rabbit IgG (Sigma Chemical Co.-Aldrich; 1:1000), FITC-conjugated goat anti-mouse $\mathrm{IgG}$ (Chemicon; 1:500), Alexa Fluor 488 goat anti-mouse IgG (Invitrogen, Carlsbad, CA; 1:1000), and Alexa Fluor 488 goat anti-rabbit IgG (Invitrogen; 1:1000) were used for secondary antibodies. The percentage of each cell type in each hemisphere was calculated. Confocal $\mathrm{Z}$ sectioning was performed to evaluate double labeling.

Statistical analysis. All data are presented as mean \pm SD. For analyzing the difference in the lesioned hemispheric volume, one-way ANOVA with the posthoc procedure was used at a 95\% significance level. The Mann-Whitney $U$ test was performed for determining the difference in the total MSC transplant counts between the sham and HI groups. It was also done to compare the total MSC counts and brain cell types between the lesioned and nonlesioned hemispheres. Neurobehavioral outcomes were compared by repeated measures of ANOVA. A $p$ value of $<0.05$ was considered statistically significant.

\section{RESULTS}

Assessment of body weight changes. The body weight of 7-d-old rats was not significantly different between the four groups before HI or sham operation (Fig. 1A). Six wk after MSC or buffer injection, the mean bodyweight of the HI-buffer group was significantly lower than that of the sham-buffer or sham-MSC group $(p=0.001$ and $p=$ 0.022 ). There was no significant difference in the body weight between the HI-buffer and HI-MSC groups ( $p=$ 0.458 , Fig. $1 B$ ).

Immunofluorescent staining. MSCs were transplanted mainly in the HI group and widely distributed to the whole brain regions $\left(793 \pm 324 / \mathrm{cm}^{2}\right.$ in the HI-MSC group, $137 \pm$ $181 / \mathrm{cm}^{2}$ in the sham-MSC group; $p=0.000$ ) (Fig. 2). Both in the sham-MSC group and HI-MSC group, the amounts of transplanted MSCs were not significantly different between the right and left hemispheres $\left(149 \pm 217 / \mathrm{cm}^{2}\right.$ versus $126 \pm$ $177 / \mathrm{cm}^{2}, p=0.732$ in sham-MSC group) or between the lesioned and nonlesioned hemispheres $\left(832 \pm 343 / \mathrm{cm}^{2}\right.$ versus $755 \pm 310 / \mathrm{cm}^{2}, p=0.865$ in the HI-MSC group) (Fig. $2 E$ ). MSCs in the sham-MSC group did not colocalize with any type of brain cells. In the HI-MSC group, they were immunopositive for all types of brain cells but usually colocalized

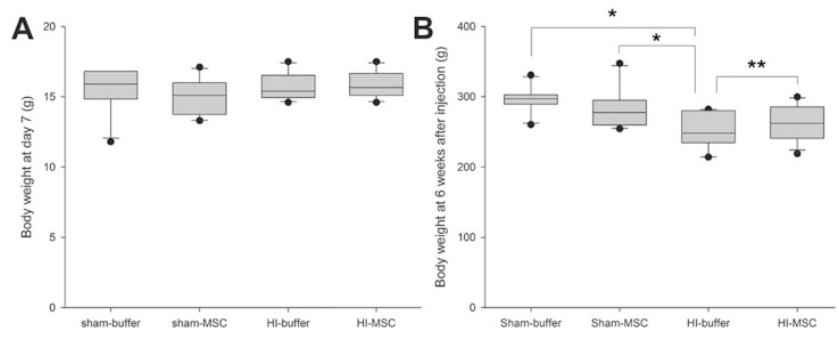

Figure 1. There was no significant difference in body weight between the study groups when the rats were 7-d-old (A) Six wk after buffer or MSC injection, the mean bodyweight was less in the HI group than in the sham group $\left({ }^{*} p<0.05\right)$. The difference was not recovered by MSC injection $(* * p=0.458)(B)$. 

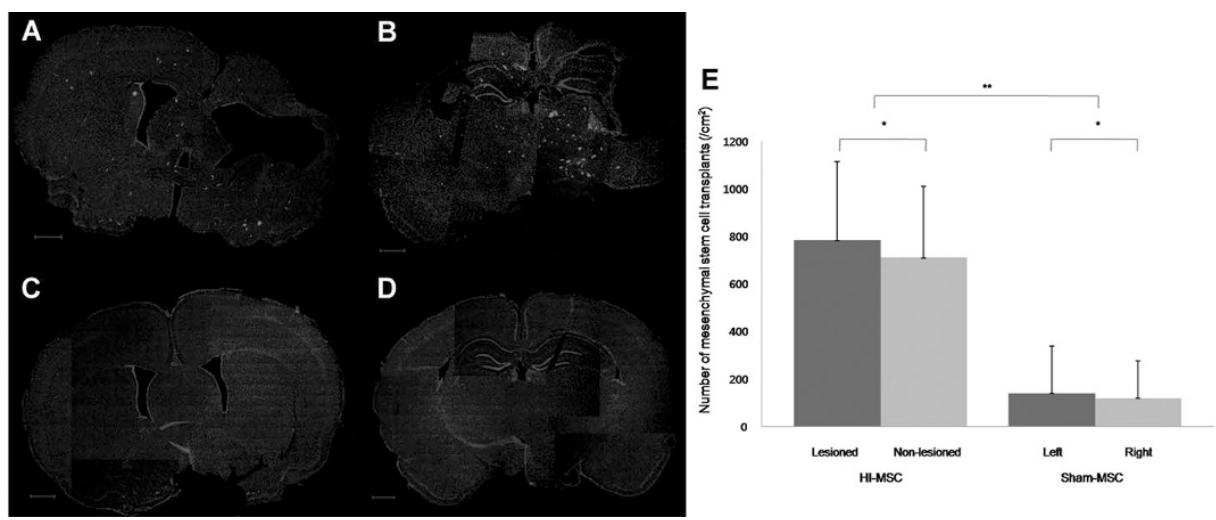

Figure 2. More MSCs were distributed throughout the entire brain $6 \mathrm{wk}$ after intracardiac injection in the HI-MSC group ( $A$ and $B)$ when compared with sham-MSC group $(C$ and $D$, original magnification $\times 200)(* * p=$ 0.000 in $E$ ). The number of $\mathrm{PKH} 26(+)$ $\mathrm{DAPI}(+)$ cells was not significantly different between the lesioned hemisphere $(n=4)$ and the nonlesioned hemisphere $(n=4)$ $(* p>0.05, E)$. Scale bar in panel $A-D$ indicates $1 \mathrm{~mm}$.
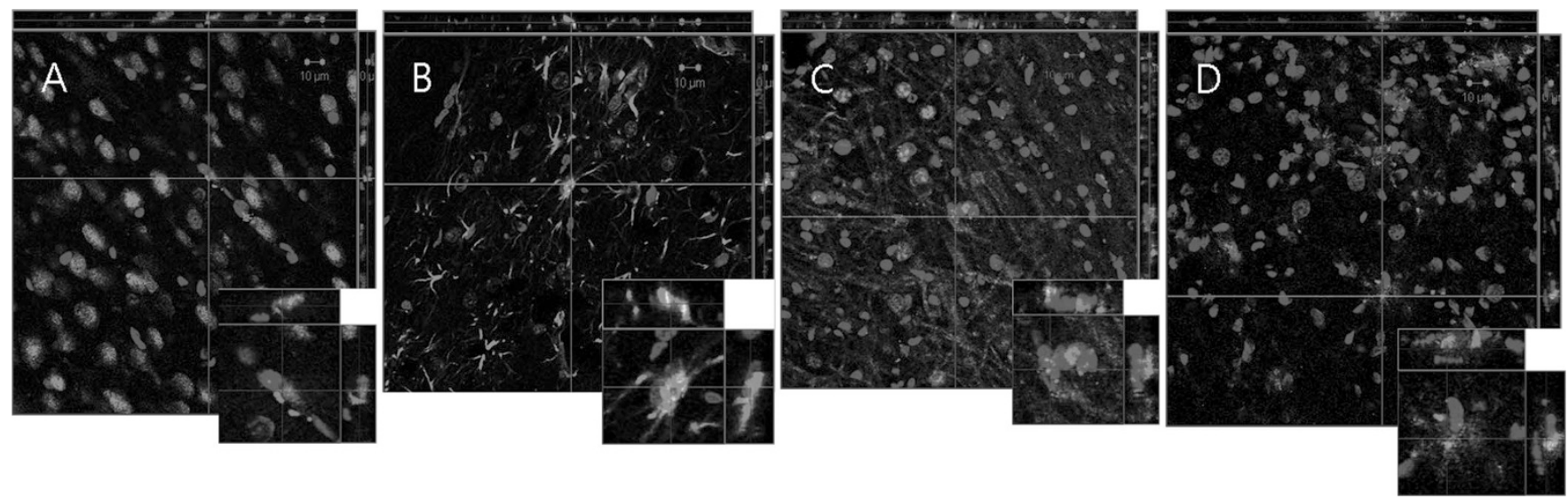

Figure 3. MSC transplants are shown with all types of brain cells including $(A)$ mature NeuN, $(B)$ GFAP, $(C)$ MBP, and $(D)$ OX42. All are shown by confocal Z-sectioning (original magnification $\times 400$ ). Scale bar indicates $10 \mu \mathrm{m}$.

Table 1. Cell types with which implanted MSCs colocalized in the rat brain following a unilateral HI injury

\begin{tabular}{lcc}
\hline Marker & $\begin{array}{c}\text { Lesioned hemisphere } \\
\text { (left) }(\%)\end{array}$ & $\begin{array}{c}\text { Nonlesioned hemisphere } \\
\text { (right) }(\%)\end{array}$ \\
\hline NeuN & $1.8 \pm 1.1$ & $1.1 \pm 0.6$ \\
GFAP & $24.8 \pm 8.6$ & $26.9 \pm 5.8$ \\
MBP & $4.2 \pm 1.8$ & $6.1 \pm 3.3$ \\
OX42 & $23.3 \pm 1.5$ & $24.9 \pm 5.1$ \\
\hline
\end{tabular}

There were no significant differences between the lesioned and nonlesioned hemispheres. Each value was expressed as mean \pm SD (\%).

with GFAP and OX42 (Fig. 3). The percentage of each brain cell type that colocalized with MSC did not significantly differ between the lesioned and nonlesioned hemispheres in the HI-MSC group (Table 1).

Cresyl violet staining. The lesioned hemisphere became atrophic after HI, and its volume was decreased to $\sim 83 \%$ of the volume of the nonlesioned contralateral hemisphere $(p=$ 0.012). MSCs administration did not improve volume of the HI injured hemisphere ( $p=0.846)$ (Fig. 4).

Neurobehavioral tests. In the cylinder test, the use of the contralateral limb was reduced in the HI-buffer group when compared with the sham-buffer group $(p=0.034)$. The use of the contralateral limb was recovered with the MSC transplants $(p=0.010)$. We also assessed neurobehavioral performance at each time point. Fourteen days after injection, the use of the contralateral limb was reduced in the HI-buffer group when

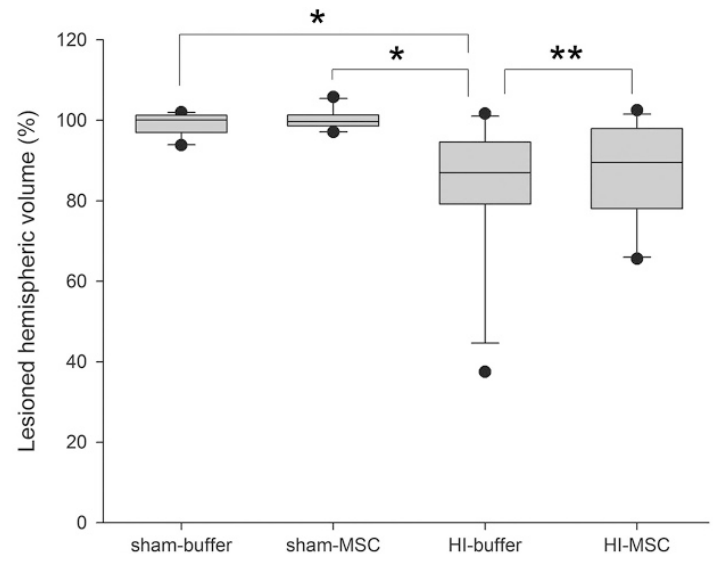

Figure 4. The percentage of the volume of the left ischemic hemisphere was compared between the four study groups. The left hemisphere became atrophic after HI, whose volume was decreased to $\sim 83 \%$ of the contralateral hemispheric volume $\left({ }^{*} p<0.05\right)$. After MSC injection, the ipsilateral hemispheric volume was not restored $(* * p>0.05)$.

compared with the sham-buffer group ( $p=0.046)$, but it was not recovered by MSC injection $(p=0.461)$. Twenty days after injection, the use of the contralateral limb was also reduced in the HI-buffer group, and it was recovered by MSC injection ( $p=0.021$ ) (Fig. 5A).

In the rotarod test, neurobehavioral performance tended to be poorer after $\mathrm{HI}$ and MSC transplant tended to improve the 

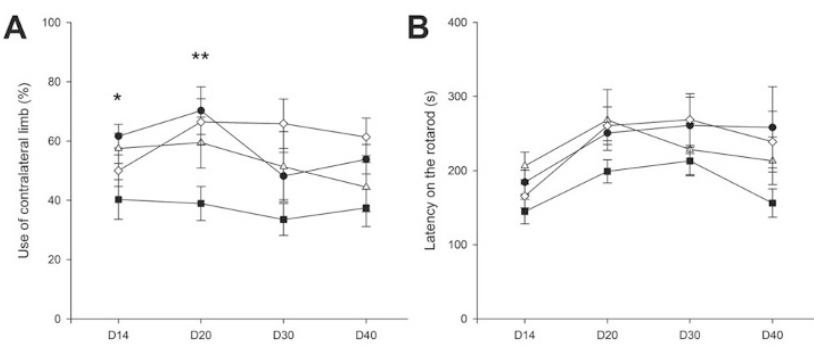

Figure 5. Neurobehavioral tests were performed. $A$, in the cylinder test, the use of the contralateral limb was reduced in the HI-buffer group when compared with the sham-buffer group ( $p=0.034)$, and it was recovered in the HI-MSC group $(p=0.010)$. When assessed at each time point, the use of the contralateral limb was reduced in the HI-buffer group when compared with the sham-buffer group $14 \mathrm{~d}$ after transplantation $(* p=0.046)$. Twenty days after transplantation, the use of the contralateral limb was also reduced in the HI-buffer group when compared with the sham-buffer group, and it was recovered in the HI-MSC group (**p $=$ 0.012 ). $B$, in the accelerating rotarod test, neurologic performance was tended to be poorer after HI. MSC transplants tended to improve the performance but this trend was not statistically significant $(p=0.184)$. $-\bullet$ - indicates sham-buffer, $-\triangle$ - indicates sham-MSC, $-\mathbf{-}-$ indicates HI-buffer, and $-\diamond-$ indicates HI-MSC.

performance, but this trend was not statistically significant $(p=0.184$, Fig. 5B).

\section{DISCUSSION}

In our study, human bone marrow-derived MSCs were significantly transplanted to the whole brain regions of the rats mainly after HI. Human MSCs gave rise to all kinds of brain cells expressing NeuN, GFAP, MBP, and OX42 after HI; however, GFAP and OX42 were more abundantly colocalized with the MSC transplants than NeuN or MBP. There were no significant differences in the total amounts of transplanted MSCs and the cell types that MSCs colocalized with between the lesioned and nonlesioned hemispheres. The volume of the lesioned hemisphere was decreased after HI, but was not restored after MSC transplantation. Neurologic performance was significantly impaired only on the cylinder test after HI, and it was improved by MSC transplants.

Our study quite differs from the previous reports, because we quantified the distribution of MSC transplants in the neonatal HI brain injury model. Another different point is that we injected the MSCs by intra-arterial injection. We also assessed the neurobehavioral outcome at a later point than the previous reports. Although MSCs could not restore the brain infarction, they showed the significant improvement in some neurobehavioral outcome.

We blindly injected MSCs into the heart instead of vein or the peritoneal cavity. The i.v. transplantation of cultured MSCs was successful in an adult rat focal ischemia model and neonatal HI model $(7,12)$. However, most MSCs can be lost in the spleen and lung via an i.v. or intraperitoneal route. Intracardiac injection is aimed at injecting them into the left ventricle of the heart to deliver more MSCs to the injured brain. Especially in neonates, the umbilical artery can be easily catheterized in a clinical situation, so the intracardiac injection method has the advantage in clinical trials.
In our study, MSCs were transplanted to the rat brain mainly after HI, and there was no significant difference in the amount of MSC transplants between the lesioned and nonlesioned hemispheres. These results are inconsistent with those of previous reports, which demonstrated that MSCs systemically infused into ischemic rats migrate preferentially to the ischemic hemisphere (7). In the neural stem cell trial in a neonatal HIE model, the amount of transplants around the ischemic boundary was much more than that of MSC transplants in our model (3). In our study, such a small amount of total transplants could not make the interhemispheric difference significant. Another explanation is that because we injected the MSCs into the left ventricle, and we ligated and cut the left common carotid artery, we could not get more MSCs in the left hemisphere because of poor perfusion even though collateral circulation. Chen et al. (7) used the intramural thread operation, and because the perfusion through the common carotid artery was intact, more MSCs could get into the ischemic hemisphere. We can perform MSC injection to the rats with carotid ligation without hypoxic exposure, and if there are less MSCs in the ligated hemisphere compared with the nonligated hemisphere, it can be due to the poor perfusion. On the other hand, because there was a very small amount of MSCs in the brain of sham-MSC group in our study, we might not be able to get a clear difference between hemispheres without hypoxia. Disruption of the blood-brain barrier may facilitate selective entry of MSCs into the brain, especially after HI. Although the newborn brain has a less effective blood-brain barrier than the adult brain, it can be more weakened by HI.

MSCs are usually differentiated into GFAP-positive cells, but few were differentiated into mature neuron. It is well established that neural stem cells and multiple classes of transit-amplifying progenitors reside in the subventricular zone and hippocampus, participating in "adult neurogenesis" (15). According to adult neurogenesis, GFAP-positive cells can represent both astrocytes and immature neural precursor cells. It will be helpful to assess the cell fate on serial time points from the earlier and later time points than $6 \mathrm{wk}$ after transplantation. Double immunofluorescent staining with Tuj1, doublecortin, or nestin is also needed to confirm that MSC was transdifferentiated into immature neural precursors.

In our study, functional improvement was independent of the reduction of brain infarction. This result suggests that although the amount of MSC transplants may not be sufficient to reduce brain infarction, it is adequate to improve functional impairments. Otherwise, it suggests that functional restoration may not be attributed to neural transdifferentiation of MSCs but that it may be a result of augmentation of neural circuits by such proteins released from grafted MSCs. Some previous studies have suggested that extracellular matrices including collagen I, fibronectin, or neurotrophic factors can enhance neuronal sprouting and synaptogenesis (16). Because MSCs are believed to secrete such extracellular matrix or neurotrophic factors, it is conceivable that MSCs or host brain cells stimulated by grafts may produce such proteins that induce functional reorganization (17). We will be able to demonstrate the increased neuronal sprouting and synaptogenesis if there is an increase of synaptophysin and GAP43 in the brain of HI-MSC group compared with HI-buffer group.

We used MSCs from bone marrow, because it can be easily acquired and cultured as compared with those from other sources including cord blood. Because it is also autotransplantable, we 
expected that one could use MSCs from their own bone marrow as cell-based therapy in a clinical condition without the problem of rejection.

The identification of MSCs is somewhat difficult, because there is no specific positive marker that can characterize MSCs. MSCs used in this study have stem cell plasticity because they can proliferate on culture with an attached fibroblast-like morphology, have a consistent set of marker proteins on their surfaces and they differentiated into neural lineages in the neonatal rat brain after $\mathrm{HI}$ as previously described $(18,19)$.

We did not use the immunosuppressive agent for these xenograft transplants because MSCs negligibly express low levels of MHC class I and do not express costimulatory molecules $(20,21)$. Liechty et al. (22) reported that even when MSCs from adult bone marrow were transplanted into fetal sheep after the expected development of immunocompetence, there was long-term engraftment. The lack of xenograft tissue rejection in the neonatal rat is also secondary to the immaturity of the neonatal immune system.

In our study, every cell was cryopreserved at first passage and thawed then recultured. Because it is very difficult to use fresh MSC every time in a clinical situation, it will be helpful if frozen and rethawed MSCs have the same stem cell plasticity as fresh MSCs. Human MSCs maintained the potential to differentiate into the osteogenic lineage for approximately up to 40 doublings on culture, even after cryopreservation (23).

We decided that the time point for our transplant would be $72 \mathrm{~h}$ after injury. At the 72-h time point, all cascades including neuronal necrosis and apoptosis are decreased, and normal energy state within the brain is restored. According to previous reports on neural stem cells, the time point between 3- and 7-d after injury was the most appropriate time for transplantation in a neonatal mouse model of HIE (24). However, we did not compare the therapeutic efficiencies of transplantation between 72-h after injury, within 24-h after injury and 7-d after injury.

We performed the cylinder test and the accelerating rotarod test because they can reveal the sensorimotor deficit caused by cortical and striatal injuries. To assess the hippocampal injury, the watermaze test is more compatible because it can assess cognitive and memory functions. In our preliminary study, our neonatal HIE model caused infarction mainly in the ipsilateral cortex, striatum, and, to a lesser degree, hippocampus. In previous studies, the cylinder test revealed a significant difference between the sham and neonatal stroke groups on the 24th postnatal day (25). Grow et al. (26) have reported that the cylinder test in P35 rat can detect sensorimotor deficits after HI.

In this study, we did not examine whether MSCs injected into the heart migrated into other organs such as the kidney, lung, spleen, liver, and heart. It is uncertain whether there may be any adverse reactions and possible tumor growth or tissue destruction because of MSC transplants. In an adult focal ischemic rat model, it was found that i.v. MSCs were detected in bone marrow up to $4 \%$ and in other organs up to $0.5 \%$, most of which encircled vessels with a few cells located in parenchyma without any adverse reactions or tumor growth (7).

In our neonatal HIE model, MSCs were transplanted and transdifferentiated to astrocytes. Although MSC transplants could not maintain the brain volume, they could improve sensorimotor deficits in the earlier period after HI. The results of this study suggest that MSC transplantation can be a new therapeutic modality for neonatal HIE, but further investigations are needed for clinical trials.

\section{REFERENCES}

1. Vannucci SJ, Hagberg H 2004 Hypoxia-ischemia in the immature brain. J Exp Biol 207:3149-3154

2. Gluckman PD, Wyatt JS, Azzopardi D, Ballard R, Edwards AD, Ferriero DM, Polin RA, Robertson CM, Thoresen M, Whitelaw A, Gunn AJ 2005 Selective head cooling with mild systemic hypothermia after neonatal encephalopathy: multicentre randomised trial. Lancet 365:663-670

3. Park KI, Hack MA, Ourednik J, Yandava B, Flax JD, Stieg PE, Gullans S, Jensen FE, Sidman RL, Ourednik V, Snyder EY 2006 Acute injury directs the migration, proliferation, and differentiation of solid organ stem cells: evidence from the effect of hypoxia-ischemia in the CNS on clonal "reporter" neural stem cells. Exp Neurol 199:156-178

4. Kim HT, Kim IS, Lee IS, Lee JP, Snyder EY, Park KI 2006 Human neurospheres derived from the fetal central nervous system are regionally and temporally specified but are not committed. Exp Neurol 199:222-235

5. Schwarz EJ, Alexander GM, Prockop DJ, Azizi SA 1999 Multipotential marrow stromal cells transduced to produce L-DOPA: engraftment in a rat model of Parkinson disease. Hum Gene Ther 10:2539-2549

6. Bjorklund A, Lindvall O 2000 Self-repair in the brain. Nature 405:892-893,895

7. Chen J, Li Y, Wang L, Zhang Z, Lu D, Lu M, Chopp M 2001 Therapeutic benefit of intravenous administration of bone marrow stromal cells after cerebral ischemia in rats. Stroke 32:1005-1011

8. Chen SL, Fang WW, Ye F, Liu YH, Qian J, Shan SJ, Zhang JJ, Chunhua RZ, Liao LM, Lin S, Sun JP 2004 Effect on left ventricular function of intracoronary transplantation of autologous bone marrow mesenchymal stem cell in patients with acute myocardial infarction. Am J Cardiol 94:92-95

9. Bang OY, Lee JS, Lee PH, Lee G 2005 Autologous mesenchymal stem cell transplantation in stroke patients. Ann Neurol 57:874-882

10. Guan XQ, Yu JL, Li LQ, Liu GX 2004 [Study on mesenchymal stem cells entering the brain through the blood-brain barrier]. Zhonghua Er Ke Za Zhi 42:920-923

11. Yasuhara T, Matsukawa N, Yu G, Xu L, Mays RW, Kovach J, Deans RJ, Hess DC, Carroll JE, Borlongan CV 2006 Behavioral and histological characterization of intrahippocampal grafts of human bone marrow-derived multipotent progenitor cells in neonatal rats with hypoxic-ischemic injury. Cell Transplant 15:231-238

12. Yasuhara T, Hara K, Maki M, Mays RW, Deans RJ, Hess DC, Carroll JE, Borlongan CV 2008 Intravenous grafts recapitulate the neurorestoration afforded by intracerebrally delivered multipotent adult progenitor cells in neonatal hypoxic-ischemic rats. J Cereb Blood Flow Metab 28:1804-1810

13. Friedenstein AJ, Gorskaja JF, Kulagina NN 1976 Fibroblast precursors in normal and irradiated mouse hematopoietic organs. Exp Hematol 4:267-274

14. Paxinos G, Watson C 2005 The Rat Brain in Stererotaxic Coordinates. 5th ed. Elsevier Academic Press, San Diego, Figures 10-60

15. Ming GL, Song H 2005 Adult neurogenesis in the mammalian central nervous system. Annu Rev Neurosci 28:223-250

16. Sakai T, Johnson KJ, Murozono M, Sakai K, Magnuson MA, Wieloch T, Cronberg T, Isshiki A, Erickson HP, Fassler R 2001 Plasma fibronectin supports neuronal survival and reduces brain injury following transient focal cerebral ischemia but is not essential for skin-wound healing and hemostasis. Nat Med 7:324-330

17. Crigler L, Robey RC, Asawachaicharn A, Gaupp D, Phinney DG 2006 Human mesenchymal stem cell subpopulations express a variety of neuro-regulatory molecules and promote neuronal cell survival and neuritogenesis. Exp Neurol 198:54-64

18. Pittenger MF, Mackay AM, Beck SC, Jaiswal RK, Douglas R, Mosca JD, Moorman MA, Simonetti DW, Craig S, Marshak DR 1999 Multilineage potential of adult human mesenchymal stem cells. Science 284:143-147

19. Li Y, Chen J, Chen XG, Wang L, Gautam SC, Xu YX, Katakowski M, Zhang LJ, Lu M, Janakiraman N, Chopp M 2002 Human marrow stromal cell therapy for stroke in rat; neurotrophins and functional recovery. Neurology 59:514-523

20. Modo M, Hopkins K, Virley D, Hodges H 2003 Transplantation of neural stem cells modulates apolipoprotein E expression in a rat model of stroke. Exp Neurol 183:320-329

21. Tse WT, Pendleton JD, Beyer WM, Egalka MC, Guinan EC 2003 Suppression of allogeneic T-cell proliferation by human marrow stromal cells: implications in transplantation. Transplantation 75:389-397

22. Liechty KW, MacKenzie TC, Shaaban AF, Radu A, Moseley AM, Deans R, Marshak DR, Flake AW 2000 Human mesenchymal stem cells engraft and demonstrate site-specific differentiation after in utero transplantation in sheep. Nat Med 6:1282-1286

23. Bruder SP, Jaiswal N, Haynesworth SE 1997 Growth kinetics, self-renewal, and the osteogenic potential of purified human mesenchymal stem cells during extensive subcultivation and following cryopreservation. J Cell Biochem 64:278-294

24. Deans RJ, Moseley AB 2000 Mesenchymal stem cells: biology and potential clinical uses. Exp Hematol 28:875-884

25. Chang YS, Mu D, Wendland M, Sheldon RA, Vexler ZS, McQuillen PS, Ferriero DM 2005 Erythropoietin improves functional and histological outcome in neonatal stroke. Pediatr Res 58:106-111

26. Grow JL, Liu YQ, Barks JD 2003 Can lateralizing sensorimotor deficits be identified after neonatal cerebral hypoxia-ischemia in rats? Dev Neurosci 25:394-402 